\title{
Spontaneous Emission by Atoms in Simple Environments
}

\author{
W. ŻAKOWICZ \\ Institute of Physics, Polish Academy of Sciences \\ al. Lotników 32/46, 02-668 Warszawa, Poland
}

\begin{abstract}
Spontaneous radiation by atoms in the presence of the planar dielectricvacuum interface, planar dielectric waveguides and cylindrical dielectric waveguides are discussed in the frame of cavity quantum electrodynamics in full analogy with that in free space. However, quantization of the electromagnetic field should be based on the modes appropriate to the selected space structure. These quantizations are usually based on incoming waves. However, the discussion of the angular intensity pattern of spontaneous emission can be simplified if the quantization is based on outgoing modes. Using these outgoing photons the angular emission radiation pattern has been obtained from a straightforward application of the perturbative method of the quantum radiation theory. Adding a contribution of the waveguiding photons attached to the waveguides (when they are present) the total emission of the spontaneous radiation and excitation decay rates of atoms radiating in these systems have been derived.
\end{abstract}

PACS numbers: $42.50 .-\mathrm{p}, 32.80 .-\mathbf{t}, 03.70 .+\mathbf{k}$

\section{Introduction}

In Purcell's brief paper [1] it has been pointed out that the spontaneous emission by an atomic system depends not only on the intrinsic atomic properties but also on the quantum properties of the electromagnetic field influenced by the configuration of material objects in the vicinity of this atom. In particular, the atom environment may have resonant features which can dramatically alter the system behaviour. For the last 20 years numerous examples of such modifications of the radiative decay rate have been experimentally shown for excited atoms near dielectric interfaces, inside resonant cavities, confocal optical resonators, and optical waveguides [2-6].

Following Purcell's idea the description of such effects in the frame of quantum theory is usually given in terms of the modified vacuum fluctuations of the 
electromagnetic field and the density of photon's states dependent on the position of the emitting atom. The quantization of the electromagnetic field and calculation of the vacuum-field fluctuations requires the knowledge of the complete set of specific electromagnetic modes existing in the selected atom environment. Nowadays all such problems are recognized as cavity quantum electrodynamics (CQED) [7].

The quantum theory offers the most fundamental and accurate description of radiation problems influenced by the environment, however, similar problems have been formulated and studied much earlier within classical electrodynamics.

Radiating antennas placed over the Earth surface have been studied by Sommerfeld since 1909 [8, 9]. His solutions describe the most important features of the influence of environment on the emission radiation pattern and total radiation power dependent on the antenna orientation and its separation from the ground. Similar effects have been shown on the atomic scale by Drexhage [10-12]. The theoretical analyses of these experiments were based on Sommerfeld's solutions [13]. Drexhage investigated also directional emission properties of atoms kept near the dielectric-vacuum interface. Though atoms and photons have been involved in these experiments, only the classical theory was applied to quantitative descriptions $[12,14]$.

Actually, the proposed quantization schemes, useful in the derivation of the environment dependent modifications to the decay rate, are not very suitable for the description of the accompanying modifications of the directional emission patterns. Since the directional emission properties are important characteristics of many optical instruments (including, e.g., lasers) the quantum formulation of the emission problem should include such possibility.

Indeed, such quantum description of these problems is possible and will be illustrated with three examples of the atom environments: (1) planar dielectricvacuum interface, (2) planar dielectric waveguide, (3) cylindrical dielectric waveguide.

\section{Spontaneous emission in free space}

Our analysis of emission properties in the presence of optical passive elements will be similar to the one developed for free space. Since the field modes used for field quantization extend over the entire infinite space, and therefore are distributions instead of normalizable functions, the basic spontaneous emission formulas are briefly summarized. It is also shown that there is no need to introduce the quantity known as the "density of photon states", which is a very important quantum object when the emission theory is based on Fermi's golden rule. The basic radiation emission formulas are based on a standard perturbation theory approach.

Free space modes of the electromagnetic field can be taken as plane waves characterized by a wave vector $\boldsymbol{k}$ and two polarization vectors $\boldsymbol{e}_{\boldsymbol{k} \mu} \mu=1,2$ orthogonal to $k$. 
Radiative properties of atoms in vacuum can be derived from

$$
\boldsymbol{E}_{T}(\boldsymbol{r}) \propto \sum_{\mu} \int \mathrm{d}_{3} k \sqrt{\omega} \boldsymbol{e}_{\boldsymbol{k} \mu}\left[\exp (\mathrm{i} \boldsymbol{k} \cdot \boldsymbol{r}) a_{\boldsymbol{k} \mu}+\exp (-\mathrm{i} \boldsymbol{k} \cdot \boldsymbol{r}) a_{\boldsymbol{k} \mu}^{\dagger}\right]
$$

with a free field Hamiltonian

$$
H_{f}=\sum_{\mu} \int \mathrm{d}_{3} k \omega a_{\boldsymbol{k} \mu}^{\dagger} a_{k \mu}, \quad H_{\mathrm{Int}}=\boldsymbol{d} \cdot \boldsymbol{E} .
$$

The perturbation theory gives the probability of emission of $\{k \mu\}$ photon

$$
P_{\boldsymbol{k} \mu}^{f} \propto \omega\left|\boldsymbol{x}_{f i} \cdot \boldsymbol{e}_{\boldsymbol{k} \mu} \exp (\mathrm{i} \boldsymbol{k} \cdot \boldsymbol{r})\right|^{2} \delta\left(\omega_{f i}-\omega(k)\right)
$$

and the radiation intensity flux into $\mathrm{d} \Omega$ spherical angle around $\hat{\boldsymbol{k}}$ direction

$$
\mathrm{d} I_{\boldsymbol{k} \mu}^{f} \propto \int_{\mathrm{d} \Omega} \mathrm{d}_{3} k \omega P_{\boldsymbol{k} \mu}^{f} \propto \omega_{f i}^{4}\left|\boldsymbol{x}_{f i} \cdot \boldsymbol{e}_{\boldsymbol{k} \mu}\right|^{2} \mathrm{~d} \Omega
$$

Integrating over all directions and summing up over two polarizations the total radiation flux is

$$
I_{\text {Total }}^{f}=\frac{e^{2}}{3 \pi \epsilon_{0}} \omega_{f i}^{4}\left|x_{f i}\right|^{2}
$$

and the radiation line or level width is

$$
\Gamma_{f i}=\frac{I_{\text {Total }}^{f}}{\omega_{f i}} .
$$

As it has been already mentioned, in this derivation of the total radiation intensity and the level width, there is no need to refer to the "density of photon states". The summation and integration over all possible photon states represented by $\sum_{\mu} \int \mathrm{d}_{3} k \ldots$ and the rules of integration of the distribution $\delta\left(\omega_{f i}-\omega(k)\right)$ incorporates the proper measure of photon states. This perturbative approach can be generalized to all environment dependent radiative processes.

\section{Environment dependent modes and field quantization}

Guided by the above description of the spontaneous emission in free space, an analogical description can be formulated for cases when the atom is surrounded by other objects. As it has already been noticed a complete set of spatial classical modes is required for the electromagnetic field quantization in a given space environment. The free space modes must be replaced by the corresponding waves fulfilling all necessary boundary conditions in the selected system. 


\subsection{Travelling in-modes and in-photons}

The first proposition of such field quantization, corresponding to the first example, has been presented by Carniglia and Mandel [15]. The plane waves composing the free space modes were replaced by triple Fresnel waves, shown in Fig. 1. Each such Fresnel triple wave is composed of an incident wave and the appropriate reflected wave propagating in the same half-space and the appropriate transmitted wave propagating in the other half-space. While the incident waves are propagating toward the interface, the reflected and transmitted waves are propagating outward.

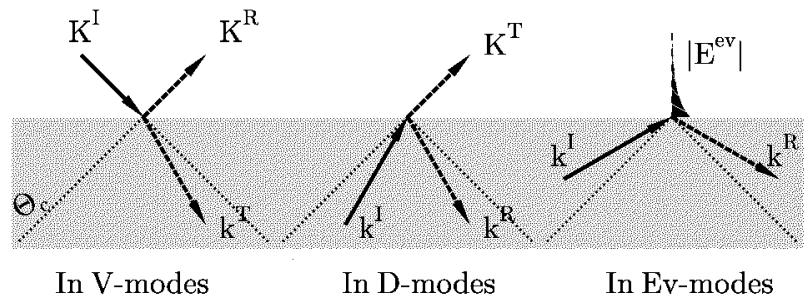

Fig. 1. Incoming modes for a planar dielectric-vacuum interface (Carniglia-Mandel modes).

These waves, forming a set of modes, are fully characterized by the incident wave, i.e. its wave vector $k$ and the incident polarization vectors $\boldsymbol{e}_{\boldsymbol{k} \mu}$. Therefore, these mode functions can be parameterized using the same vectors as the free-space modes. The amplitudes of the reflected and transmitted waves are uniquely determined by the field continuity conditions, and are known as Fresnel coefficients.

For Fresnel waves (1)

$$
\mathcal{E}_{\boldsymbol{k} \mu}=\left\{\begin{array}{l}
\text { incident }+ \text { reflected }, \\
\text { transmitted. }
\end{array}\right.
$$

The incident wave $\boldsymbol{e}_{\boldsymbol{k} \mu} \exp (\mathrm{i} \boldsymbol{k} \cdot \boldsymbol{r})$ can arrive either from the vacuum or dielectric side and thus the vacuum or dielectric photons can be distinguished. Accordingly, there are different relations between the length of $|k|$ and the wave frequency $\omega(k)$

$$
\omega(k)= \begin{cases}k, & \text { for vacuum photons } \\ k / n, & \text { for dielectric photons. }\end{cases}
$$

Among the dielectric modes the evanescent waves for which $k_{\|}>\omega$ (not possible in vacuum) can be distinguished. They are also known as the waves with total internal reflected at a boundary. Their magnitudes in the vacuum half-space are exponentially decreasing with a growing distance from the interface.

It has been shown in [16] that all these waves or modes form a complete set and thus can be used for the electromagnetic field quantization. 
In the cases (2) and (3) in which the space is modified by the presence of dielectric waveguides, either planar (2) or cylindrical (3), the appropriate modes may be chosen as solutions for the corresponding scattering problem. Starting from the incident plane wave $\boldsymbol{e}_{\boldsymbol{k} \mu} \exp (\mathrm{i} \boldsymbol{k} \cdot \boldsymbol{r})$ and solving the scattering problem, the mode parameterized by vectors $\boldsymbol{k}$ and $\boldsymbol{e}_{\boldsymbol{k} \mu}$ can be represented as

$$
\mathcal{E}_{\boldsymbol{k} \mu}= \begin{cases}\boldsymbol{e}_{k \mu} \exp (\mathrm{i} k \cdot \boldsymbol{r})+\text { scattered wave, } & \text { outside waveguide } \\ \text { internal field }, & \text { inside waveguide }\end{cases}
$$

(scattered waves are directed outwards). The exact forms of such modes are presented in [17-21]. Using the above modes, named "travelling" as they represent the waves extended over the entire space, in the quantization procedure, one may introduce the corresponding "travelling photons" and write the relevant part of the electric field operator $\mathcal{E}_{\boldsymbol{k} \mu}(\boldsymbol{r})$ in the form

$$
\boldsymbol{E}_{T}(r) \propto \sum_{\mu} \int \mathrm{d}_{3} k \sqrt{\omega(k)}\left[\mathcal{E}_{k \mu}(r) a_{\boldsymbol{k} \mu}+\mathcal{E}_{\boldsymbol{k} \mu}^{\star}(r) a_{\boldsymbol{k} \mu}^{\dagger}\right]
$$

\subsection{Waveguided modes and photons}

The above travelling waves do not form a complete set of propagating modes. They must be supplemented by the waves attached to and propagating along the waveguide (waveguided waves). An example of such waves is presented in Fig. 2.

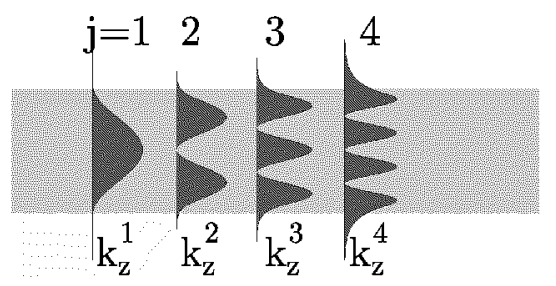

Fig. 2. Sketch of waveguided modes for a planar dielectric waveguide.

For any value of the longitudinal wave vector (either $k_{\|}$in (2) or $k_{z}$ in (3)) there is only a discrete set of the possible frequencies $\omega^{i}\left(k_{z}\right)$. Thus, the part of the electric field operator dependent on the waveguided modes and photons is

$$
\boldsymbol{E}_{W}(\boldsymbol{r}) \propto \sum_{i} \int \mathrm{d} k_{z} \sqrt{\omega^{i}\left(k_{z}\right)}\left[b_{k_{z}}^{i} \mathcal{E}_{k_{z}}^{i}(\boldsymbol{r})+b_{k_{z}}^{i \dagger} \mathcal{E}_{k_{z}}^{* i}(\boldsymbol{r})\right],
$$

and the waveguiding photon contribution to the free field Hamiltonian is

$$
H_{w}=\sum_{i} \int \mathrm{d} k_{z} \omega^{i}\left(k_{z}\right) b_{k_{z}}^{\dagger i} b_{k_{z}}^{i} .
$$

For the waveguiding waves $\omega^{i}\left(k_{z}\right) / k_{z}<c$. 
The travelling waves together with the waveguiding waves form an orthogonal system of modes (perhaps complete) can be used for the field quantization and the perturbation theory of radiation. The quantized field has been used for calculations of the vacuum fluctuations and the density of photon's states dependent on the position and the actual structure of the system. These results together with Fermi's golden rule were fundamental for the estimations of the atom emission rates dependent on its position and orientation $[17,18,22,6]$.

However, not only the global decay rates can be measured but also the angular characteristic of the emitted radiation is a measurable quantity, e.g. $[11,12,14]$. In these former discussions the angular patterns have been calculated according to the classical electrodynamics, although Drexhage's experiments involved atoms and photons.

First, it must be noticed that the travelling photons based on the incoming modes are not very convenient for such quantum description of the emission pattern [23]. It can be found that many such photons may contribute to the radiation emitted in a given direction. So, if a direction sensitive photodetector is placed, e.g. in vacuum side, it can be affected by the transmitted part of dielectric modes and the reflected part of vacuum modes (propagating toward the detector). That detector cannot distinguish between the two different photons, parameterized by the incident waves. Therefore, the two incident waves, coming from the opposite sides of the interface, do interfere and the amplitudes of the emission of both photons must be added coherently [24].

The alternative derivation of the radiation emission pattern, and holding the same in-photons, has been proposed in [25]. This indirect method, which the authors describe as simple (the opinion not shared by me), is based on Lorentz's reciprocity theorem applied to the inverse process of a photon absorption.

However, directional properties of the spontaneous emission can be studied in a more direct way by another choice of modes in the field quantization, and consequently using a different base for the photon states.

\subsection{Travelling out-modes and out-photons}

The travelling solutions of Maxwell equations are highly degenerated. Therefore, besides the modes based on the incident waves there are other sets of the travelling modes possible.

It has been shown in [19-21] that for the quantum description of radiation emission problems, it is more suitable to perform the field quantization with the outgoing modes instead of those based on the incoming modes. The outgoing modes can be parameterized by the emerging waves similarly as the incoming modes were parameterized by the incident waves. The emerging waves are propagating outward the interface in (1) or the waveguides in (2) and (3) as plane waves and thus can also be parameterized by $k$ and $\boldsymbol{e}_{\boldsymbol{k} \mu}$. In order to get the outgoing modes, these plane waves must be supplemented by the assembling waves propagating 


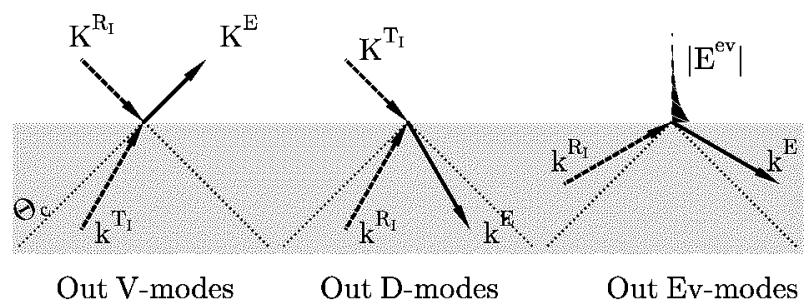

Fig. 3. Outgoing modes for a planar dielectric-vacuum interface.
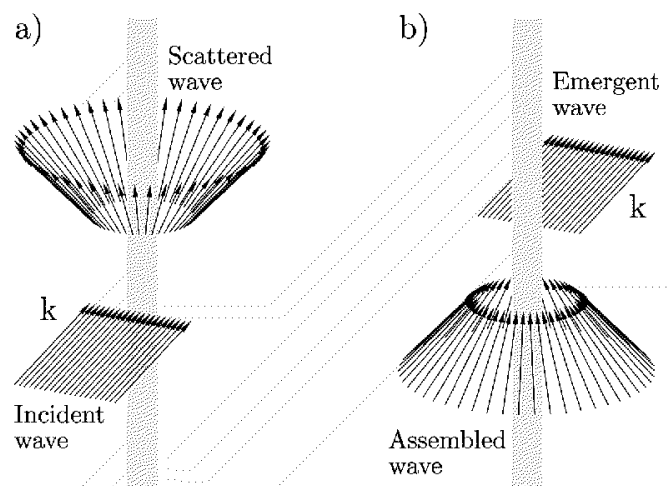

Fig. 4. In- (a) and out- (b) travelling modes for a dielectric circular waveguide.

toward the interface. As can be seen in Figs. 3 and 4 only one outgoing photon contributes to the photon measurement in a given direction.

Let me stress that both systems of the travelling modes incoming or outgoing are equivalent and each of them can be expressed in terms of the other. So, any radiative process can be described in both forms of the quantized field. Nevertheless, the description of the emission processes is simpler in terms of the outgoing photons because the problem of interference among different photons has been eliminated. On the other hand, the incoming photons are more suitable for the description of absorption processes.

Therefore, an alternative mode functions, based on outgoing functions, is proposed.

Thus, instead of Fresnel waves (1), one can take

$$
\mathcal{E}_{\boldsymbol{k} \mu}^{\text {out }}=\left\{\begin{array}{l}
\text { emergent }+ \text { in-reflected } \\
\text { in-transmitted }
\end{array}\right.
$$

and for the cases (2) and (3) 


$$
\mathcal{E}_{\boldsymbol{k} \mu}^{\text {out }}= \begin{cases}\boldsymbol{e}_{\boldsymbol{k} \mu} \exp (\mathrm{i} \boldsymbol{k} \cdot \boldsymbol{r})+\text { assembled wave, } & \text { outside waveguide } \\ \text { internal field, } & \text { inside waveguide }\end{cases}
$$

(assembled waves are directed inward). Travelling photon emission (angular emission pattern)

$$
\frac{\mathrm{d} I_{\boldsymbol{k} \mu}}{\mathrm{d} \Omega} \propto \frac{1}{\Delta \Omega} \int_{\Delta \Omega} \mathrm{d}_{3} k \omega P_{\boldsymbol{k} \mu} \propto \omega^{4}\left|\boldsymbol{x}_{f i} \cdot \mathcal{E}_{k \mu}^{\text {out }}\left(\boldsymbol{R}_{a}\right)\right|^{2} .
$$

The total power of radiation emitted to the outer space can be obtained by the integration of $\mathrm{Eq}$. (13) over all photon directions and the summation over its polarizations.

Waveguided photon emission (discrete mode excitations)

$$
I_{i} \propto\left(\left|\frac{\partial \omega^{i}\left(k_{z}\right)}{\partial k_{z}}\right|_{k_{z}^{i}}\right)^{-1}\left|x_{f i} \cdot \mathcal{E}_{k_{z}^{i}}^{i}\left(\boldsymbol{R}_{a}\right)\right|^{2} .
$$

Let me point out a dependence of the emission properties on the value of the electric field of the mode at the position of the atom $\boldsymbol{R}_{a}$. In the case of the waveguided modes notice a dependence of $I_{i}$ on the group velocity of the mode $v_{\mathrm{gr}}^{i}=\partial \omega^{i}\left(k_{z}\right) / \partial k_{z}$. In order to find the contribution of the waveguiding photons to the emission rates, $I_{i}$ must be summed up over all possible waveguided modes. For a given frequency of photons, determined by the atomic transition, there is only a finite number of these photons. Therefore,

$$
I_{\text {Total }}\left(\boldsymbol{R}_{a}\right)=\sum_{\mu=1,2} \int \mathrm{d} \Omega \frac{\mathrm{d} I_{\mathbb{k}_{k} \mu}}{\mathrm{d} \Omega}+\sum_{i} I_{i}
$$

This formula replaces Eq. (5) valid in the empty space.

It is convenient to normalize the emission intensities and rates

$$
\Gamma\left(\boldsymbol{R}_{a}\right)=\frac{I_{\text {Total }}\left(\boldsymbol{R}_{a}\right)}{I_{\text {Total }}^{f}} .
$$

Several examples taken from [19-21] are illustrating the above discussion and formulae.

\section{Examples}

The first two examples (Fig. 5a and b) show the radiation pattern for a dipole kept in the vicinity of a dielectric-vacuum interface at various distances from that interface. Notice that the dipole placed in vacuum very close to the interface emits significantly evanescent photons. The emission patterns for dipoles in vacuum, taken from [19], are identical with those obtained using Lorentz's reciprocity theorem presented in [25]. The program used to compute the radiation pattern for the dipole in the dielectric had a small error, hence Fig. 5 in [19] is incorrect and should be replaced by Fig. 5a of the present paper. 
a)

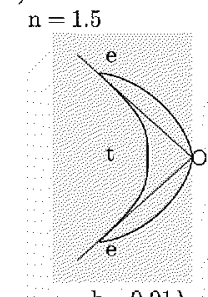

$h=-0.01 \lambda$

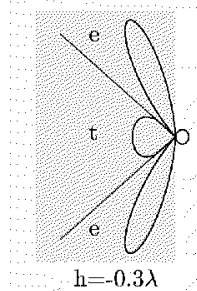

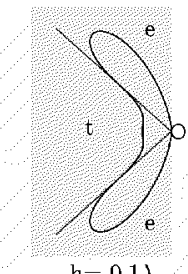

$\mathrm{h}=-0.1 \lambda$

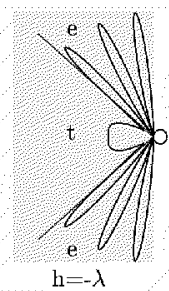

b)
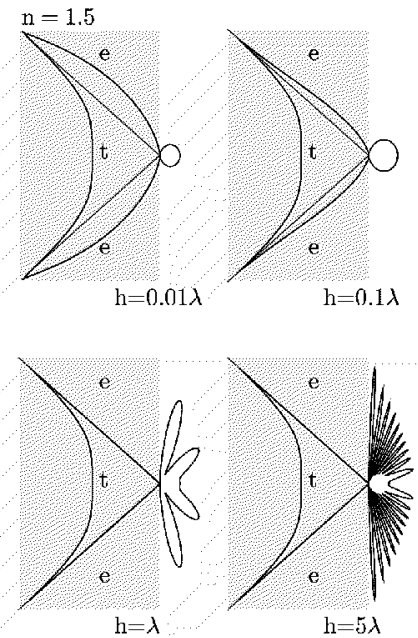

Fig. 5. Radiation intensity pattern for a dipole oscillating parallel to the dielectricvacuum interface for several dipole's positions: (a) dipole in dielectric, (b) dipole in vacuum. The radiation is observed in a plane normal to the interface and directed along the dipole.

a)

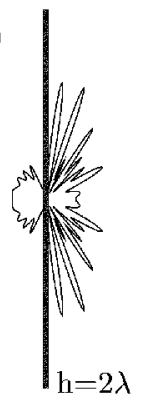

b)

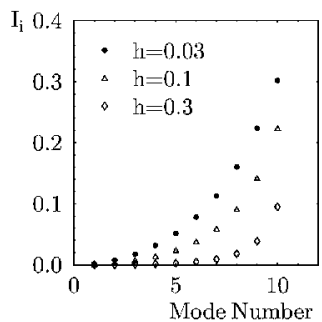

c)

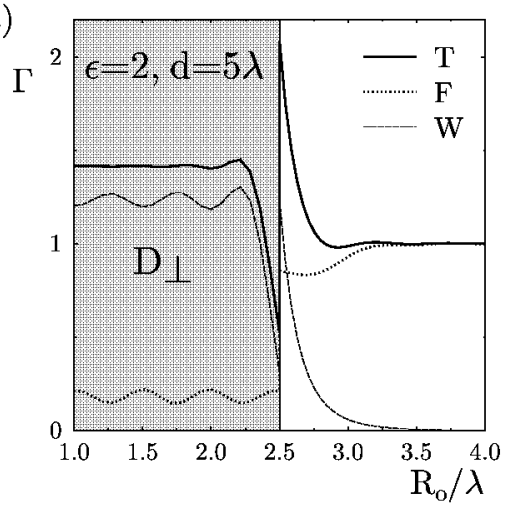

Fig. 6. Radiation intensity pattern (a), waveguided photons excitation (b), and total radiation intensity (c) for a dipole in the vicinity of a planar waveguide.

Figure 6 illustrates the properties of a dipole radiation in the vicinity of a planar dielectric waveguide. They include the travelling photons contributing to the radiation intensity angular pattern (a), excitation of the waveguiding photons (b), and the global radiative decay as a function of the dipole position. It is worth pointing out that the fastest decay corresponds to the dipoles placed just outside the dielectric surface and is due to the emission of the waveguiding photons. In 
a)

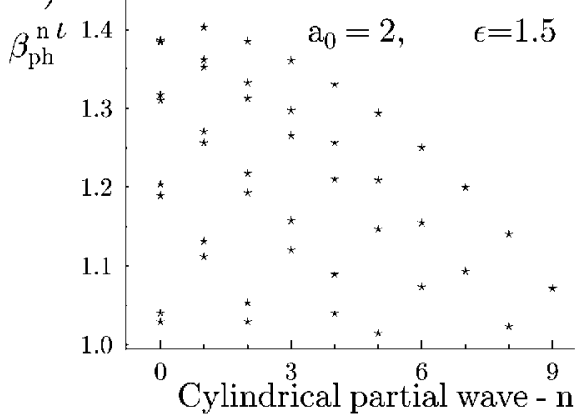

b)

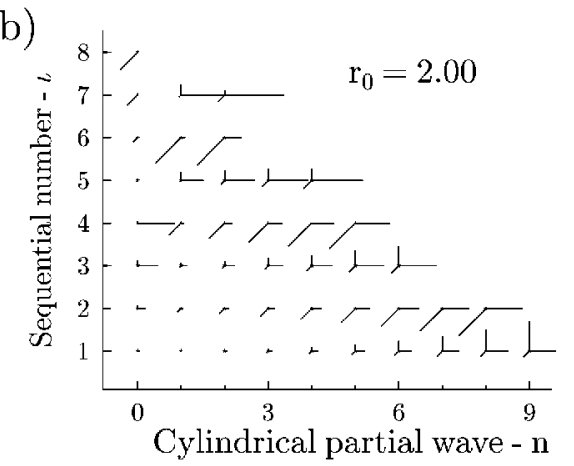

Fig. 7. Cylindrical dielectric waveguide: (a) $\beta_{p h}=k_{z} / \omega$ for $E_{n, \iota}$ waveguiding modes, (b) excitations of waveguiding modes represented by the length of the lines for a dipole oscillating along either $\boldsymbol{e}_{r}$ (horizontal), or $\boldsymbol{e}_{z}$ (vertical), or $\boldsymbol{e}_{\phi}$ (skew).
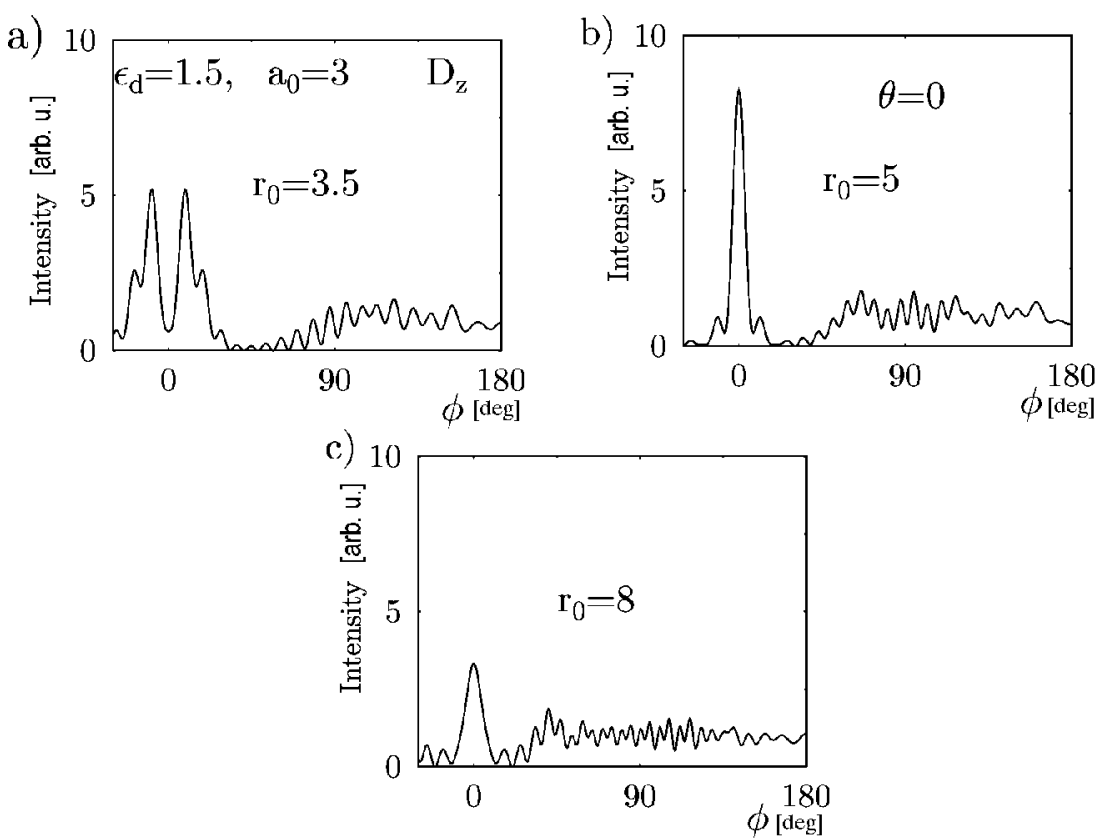

Fig. 8. Radiation intensity angular characteristics in a plane orthogonal to the circular dielectric waveguide for different dipole positions: (a) $r_{0}=3.5\left(r_{0}<d_{\mathrm{F}}\right)$, (b) $r_{0}=5$ $\left(r_{0}=d_{\mathrm{F}}\right)$, and $(\mathrm{c}) r_{0}=8\left(r_{0}>d_{\mathrm{F}}\right)\left(d_{\mathrm{F}}-\right.$ focal length $)$.

a similar way in Figs. 7, 8, and 9 the emission features for dipoles in the vicinity of a dielectric cylinder are characterized. The modes are parameterized by the cylindrical partial wave number $n$ and the sequential number in each class of the partial waves $\iota$. The excitation of the individual modes by a dipole oriented along the $\boldsymbol{e}_{r}, \boldsymbol{e}_{z}$, and $\boldsymbol{e}_{\phi}$ directions is proportional to the length of the corresponding 

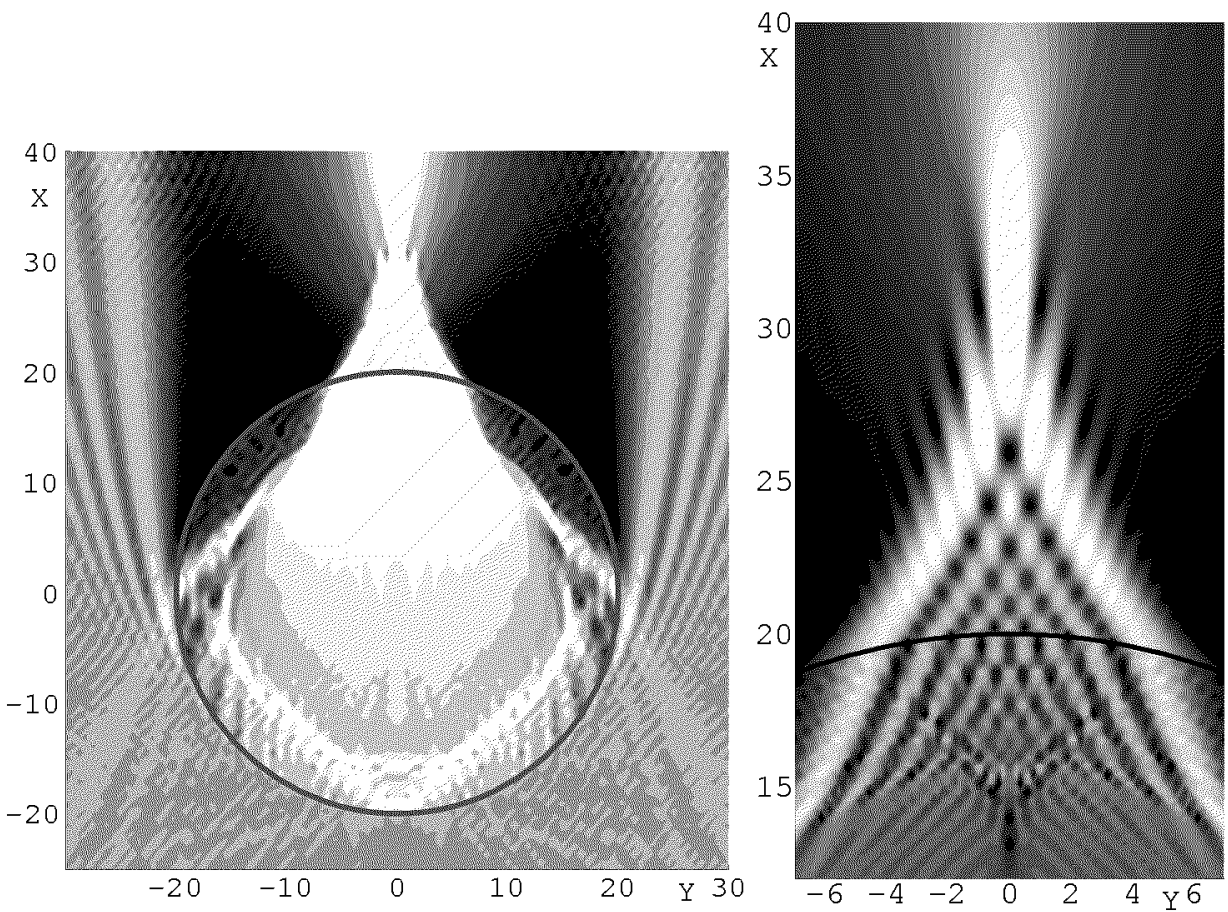

Fig. 9. Field mode in the presence of a dielectric cylinder represented by the magnitude of the electric field in a horizontal plane orthogonal to a cylinder; a general view (left) and the focal region (right).

a)

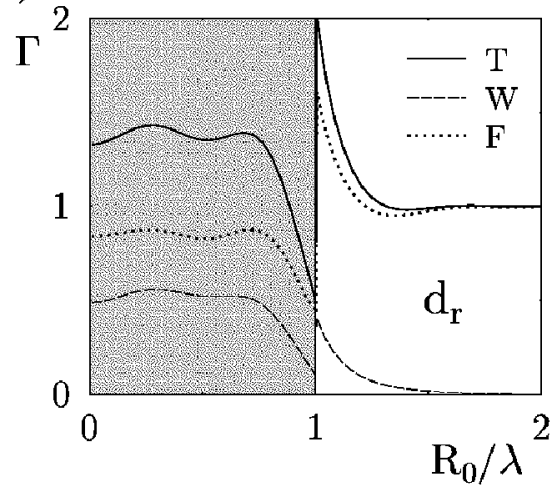

b)

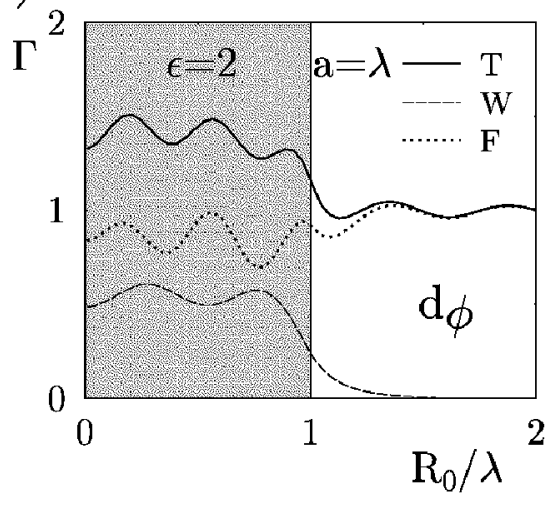

Fig. 10. Dipole excitation decay rate in the presence of a circular dielectric waveguide as a function of the dipole position; (a) dipole along $\boldsymbol{e}_{r}$ and (b) dipole along $\boldsymbol{e}_{\phi}$.

horizontal, vertical, and skew lines.

The angular radiation patterns shown in Fig. 8 reflect the focusing properties 
of the mode scattered (or assembled) by a dielectric cylinder acting like a lens. The double peak, single sharp peak, and single spread peak correspond to: the dipole placed between the cylinder and the focal point (a), at the focal point (b) and farther away from the focal point. More properties of this focused mode are shown in Fig. 9a and b. They include a detail structure of the focused wave which in optics is usually computed using various approximate diffraction theories and here it is based on the almost exact solution of Maxwell equations. More on that can be found in [26]. Although the radiation emission pattern is a very complex and rapidly changing function of the dipole position, the global decay rates, shown in Fig. 10, behave more regularly similar to the previous case of planar dielectric waveguide.

\section{Final remarks}

This discussion and the presented examples show that the spontaneous emission by atoms placed in the vicinity of other objects influencing the structure and the properties of the electromagnetic field can be adequately described using the standard perturbative methods of the quantum radiation theory. It is only required that the quantization of the electromagnetic field is performed in accordance with the structure of atoms' environment. A complete set of modes and associated with them photons include travelling photons, with the properties analogical to the photons in the empty space, and the waveguiding photons. The waveguiding photons, absent in the empty space, are very important for the emission by atoms inside and very close to the waveguide.

In almost all papers discussing environment effects in the spontaneous emission, the modifications are attributed to the changes in the quantum vacuum-field fluctuations, and the changes in the density of photon states.

However, the density of photo's states depends on the selected parameterization of the field modes of the system. With the parameterization of the travelling photons used in this paper the density of travelling photon states is exactly the same as in free space. Therefore, it is more important that the magnitude of the vacuum-fields (or the modes) is position dependent. Thus the coupling between the corresponding photons and the excited dipole becomes dependent on the dipole's position. In consequence all radiative processes for this dipole are modified.

The understanding of radiative processes of atoms placed in a particular environment is important not only for studies of fundamental principles but also for practical applications in many photonic instruments, including, e.g., various lasers (ordinary and semiconducting), photo detectors, and photo amplifiers, etc. The performance of all these instruments depends on a proper influence on the radiative processes of atoms.

\section{References}

[1] E.M. Purcell, Phys. Rev. 69, 681 (1946).

[2] R.G. Hulet, E.S. Hilfer, D. Kleppner, Phys. Rev. Lett. 55, 2137 (1985). 
[3] W. Jhe, A. Anderson, E.A. Hinds, D. Meschede, L. Moi, S. Haroche, Phys. Rev. Lett. 58, 666 (1987).

[4] D.J. Heinzen, M.S. Feld, Phys. Rev. Lett. 59, 2623 (1987).

[5] E. Yablonovich, T.J. Gmitter, R. Bhat, Phys. Rev. Lett. 61, 2546 (1988).

[6] E. Snoeks, A. Lagendijk, A. Polman, Phys. Rev. Lett. 74, 2459 (1995).

[7] S. Haroche, D. Kleppner, Physics Today, January, 24 (1989).

[8] A. Sommerfeld, Ann. d. Phys. 28, 665 (1909).

[9] A. Sommerfeld, Partial Differential Equations in Physics, Academic Press, New York 1949.

[10] K.H. Drexhage, J. Lumin. 1-2, 693 (1970).

[11] K.H. Drexhage, in: Progress in Optics, Ed. E. Wolf, Vol. XII, North-Holland, Amsterdam 1974, p. 165.

[12] C.K. Carniglia, L. Mandel, K.H. Drexhage, J. Opt. Soc. Am. 62, 479 (1972).

[13] H. Morawitz, Phys. Rev. 187, 1792 (1969).

[14] W. Lukosz, R.A. Kunz, J. Opt. Soc. Am. 67, 1607 and 1615 (1977); W. Lukosz, ibid. 69, 1495 (1979).

[15] C.K. Carniglia, L. Mandel, Phys. Rev. D 3, 280 (1971).

[16] I. Białynicki-Birula, J.B. Brojan, Phys. Rev. D 5, 485 (1972).

[17] H. Khosravi, R. Loudon, Proc. R. Soc. Lond. A 433, 337 (1991).

[18] H. Khosravi, R. Loudon, Proc. R. Soc. Lond. A 436, 373 (1992).

[19] M. Janowicz, W. Żakowicz, Phys. Rev. A 50, 4350 (1994).

[20] W. Żakowicz, A. Błędowski, Phys. Rev. A 52, 1640 (1995).

[21] W. Żakowicz, M. Janowicz, Phys. Rev. A 62, 013820 (2000).

[22] H.P. Urbach, G.L.J.A. Rikken, Phys. Rev. A 57, 3913 (1998).

[23] R.J. Glauber, M. Lewenstein, Phys. Rev. A 43, 467 (1991).

[24] W. Żakowicz, Phys. Rev. A 52, 882 (1995).

[25] J.-Y. Courtois, J.-M. Courty, J.C. Mertz, Phys. Rev. A 53, 1862 (1996).

[26] W. Żakowicz, Phys. Rev. E 64, 066610 (2001). 